PROCEEDINGS OF THE

AMERICAN MATHEMATICAL SOCIETY

Volume 127, Number 12, Pages 3623-3631

S 0002-9939(99)05121-7

Article electronically published on May 17, 1999

\title{
DIMENSION OF THE GLOBAL ATTRACTOR FOR DAMPED NONLINEAR WAVE EQUATIONS
}

\author{
ZHOU SHENGFAN
}

(Communicated by Michael Handel)

\begin{abstract}
An estimate on the Hausdorff dimension of the global attractor for damped nonlinear wave equations, in two cases of nonlinear damping and linear damping, with Dirichlet boundary condition is obtained. The gained Hausdorff dimension is bounded and is independent of the concrete form of nonlinear damping term. In the case of linear damping, the gained Hausdorff dimension remains small for large damping, which conforms to the physical intuition.
\end{abstract}

\section{INTRODUCTION}

Consider the nonlinear wave equation, with nonlinear damping,

$$
u_{t t}+h\left(u_{t}\right)-\Delta u+f(u)=g, x \in \Omega, t>0,
$$

with the Dirichlet boundary condition

$$
\left.u(x, t)\right|_{x \in \partial \Omega}=0, t>0,
$$

and the initial value conditions

$$
u(x, 0)=u_{0}(x), \frac{\partial u}{\partial t}(x, 0)=u_{1}(x), x \in \Omega,
$$

where $u=u(x, t)$ is a real-valued function on $\Omega \times[0,+\infty), \Omega$ is an open bounded set of $R^{n}$ with a smooth boundary $\partial \Omega, D(-\triangle)=H_{0}^{1}(\Omega) \cap H^{2}(\Omega), g \in L^{2}(\Omega)$.

We assume conditions on $f(u)$ and $h(v)$ as follows:

(i) $f(u) \in C^{2}(R ; R)$ satisfies:

(4) $f(0)=0, \lim _{|u| \rightarrow+\infty} \sup \frac{f(u)}{u} \leq 0,\left|f^{\prime}(u)\right| \leq k,\left|f^{\prime}\left(u_{1}\right)-f^{\prime}\left(u_{2}\right)\right| \leq k_{1}\left|u_{1}-u_{2}\right|^{\delta_{1}}$,

for any $u, u_{1}, u_{2} \in R$, where $k, k_{1}, \delta_{1}>0$.

(ii) $h(v) \in C^{1}(R ; R)$ satisfies:

$$
h(0)=0,0<\alpha \leq h^{\prime}(v) \leq \beta<+\infty,\left|h^{\prime}\left(v_{1}\right)-h^{\prime}\left(v_{2}\right)\right| \leq k_{2}\left|v_{1}-v_{2}\right|^{\delta_{2}},
$$

for any $v, v_{1}, v_{2} \in R$, where $k_{2}, \delta_{2}>0$.

Hale [1] presented the existence and uniqueness of solution of system (1)-(3) which defines a continuous semigroup of mapping $S(t):\left\{u_{0}, u_{1}\right\} \mapsto\left\{u, u_{t}\right\}$, for $t \geq o$, from $E=H_{0}^{1}(\Omega) \times L^{2}(\Omega)$ into itself, and also showed that there is a global

Received by the editors February 19, 1998.

1991 Mathematics Subject Classification. Primary 35B40, 35L70.

Key words and phrases. Wave equation, global attractor, Hausdorff dimension.

This research was supported by the National Natural Science Foundation of China. 
attractor $B$ in $E$ for (1)-(3). If the damping is linear, i.e., the function $h(v)=\alpha v$ in which $\alpha>0$, Temam [2] gave an upper bound of the Hausdorff dimension of the attractor, but this upper bound is directly proportional to the coefficient $\alpha$ of damping for large $\alpha$ and tends to infinity as $\alpha \rightarrow+\infty$, which does not conform to the physics.

In this paper, we obtain an upper bound of the Hausdorff dimension for the global attractor $B$ for system (1)-(3), in two cases of nonlinear damping and linear damping, by carefully estimating and splitting the positivity of the linear operator in the corresponding evolution equation of the first order in time. The idea of using such a technique was originated by $\mathrm{G}$. Wang and S. Zhu [3]. The gained Hausdorff dimension is bounded and is independent of the concrete form of the nonlinear damping term $h(v)$. Particularly, for the linear damping, the Hausdorff dimension of the attractor remains small for large damping, which improves the estimate given by Temam. The main results are the following theorems.

Theorem 1. For system (1)-(3) with nonlinear damping. Let

$$
\begin{gathered}
\gamma=\frac{2\left(\beta^{2}+4 \lambda_{1}\right)}{4\left(\beta^{2}+4 \lambda_{1}\right)+\left(\sqrt{\frac{8}{27} \beta^{3}+4 \lambda_{1}^{2} \alpha^{2}}+2 \lambda_{1} \alpha\right)^{\frac{2}{3}}+\left(\frac{8}{27} \beta^{3}+4 \lambda_{1}^{2} \alpha^{2}\right)^{\frac{1}{3}}+\frac{2}{3} \beta}, \\
\sigma=\frac{4 \lambda_{1} \gamma^{2} \alpha}{\beta^{2}+4 \lambda_{1}+\sqrt{\left(\beta^{2}+4 \lambda_{1}\right)\left(\beta^{2}+4 \lambda_{1}-16 \gamma^{2} \lambda_{1}\right)}} .
\end{gathered}
$$

Then the Hausdorff dimension $d_{H}(B)$ of the global attractor $B$ in $E$ satisfies:

$$
d_{H}(B) \leq \min \left\{\ell \mid \ell \in N, \frac{1}{\ell} \sum_{j=1}^{\ell} \lambda_{j}^{-1} \leq \frac{2 \alpha \sigma}{k^{2}}\right\}
$$

where $\left\{\lambda_{j}\right\}_{j \in N}, 0<\lambda_{1} \leq \lambda_{2} \leq \cdots \leq \lambda_{m} \leq \cdots$, are the eigenvalues of operator $-\Delta$ with the Dirichlet boundary condition on $\Omega$.

It is easy to see from $(7)$ that $d_{H}(B)$ is bounded because

$$
\frac{1}{\ell} \sum_{j=1}^{\ell} \lambda_{j}^{-1} \rightarrow 0
$$

as $\ell \rightarrow+\infty$.

Theorem 2. For system (1)-(3) with linear damping $h(v)=\alpha v$. The Hausdorff dimension $d_{H}\left(B_{1}\right)$ of the global attractor $B_{1}$ satisfies:

$$
d_{H}\left(B_{1}\right) \leq \min \left\{\ell \mid \ell \in N, \frac{1}{\ell} \sum_{j=1}^{\ell} \lambda_{j}^{-1} \leq \frac{2 \lambda_{1} \alpha^{2}}{k^{2} \sqrt{\alpha^{2}+4 \lambda_{1}}\left(\alpha+\sqrt{\alpha^{2}+4 \lambda_{1}}\right)}\right\} .
$$

Obviously, $d_{H}\left(B_{1}\right)$ in (8) remains small for sufficiently large damping $\alpha$ because

$$
\frac{2 \lambda_{1} \alpha^{2}}{k^{2} \sqrt{\alpha^{2}+4 \lambda_{1}}\left(\alpha+\sqrt{\alpha^{2}+4 \lambda_{1}}\right)} \rightarrow \frac{\lambda_{1}}{k^{2}}
$$

as $\alpha \rightarrow+\infty$. 


\section{Preliminaries}

In this section, we present the existence and uniqueness of solutions, and the existence of the global attractor for problem (1)-(3).

It is known that the operator $A=-\Delta: D(A) \rightarrow L^{2}(\Omega)$ is a self-adjoint positive linear operator and its eigenvalues $\left\{\lambda_{i}\right\}_{i \in N}$ satisfy

$$
0<\lambda_{1} \leq \lambda_{2} \leq \cdots \leq \lambda_{m} \leq \cdots, \lambda_{m} \rightarrow+\infty(m \rightarrow+\infty) .
$$

Let

$$
\begin{gathered}
(u, v)=\int_{\Omega} u v d x,|u|=(u, u)^{\frac{1}{2}}, \forall u, v \in L^{2}(\Omega), \\
((u, v))=\int_{\Omega} \nabla u \cdot \nabla v d x,\|u\|=((u, u))^{\frac{1}{2}}, \forall u, v \in H_{0}^{1}(\Omega),
\end{gathered}
$$

and

$$
\begin{gathered}
\left(y_{1}, y_{2}\right)_{E}=\left(\left(u_{1}, u_{2}\right)\right)+\left(v_{1}, v_{2}\right), \forall y_{i}=\left(u_{i}, v_{i}\right)^{T} \in E, i=1,2, \\
|y|_{E}=(y, y)_{E}^{\frac{1}{2}}, \forall y=(u, v)^{T} \in E
\end{gathered}
$$

denote the usual inner products and norms in $L^{2}(\Omega), H_{0}^{1}(\Omega)$ and $E=H_{0}^{1}(\Omega) \times L^{2}(\Omega)$ respectively.

Let $u_{t}=v$; then (1)-(3) are equivalent to the following initial value problem in the Hilbert space $E$ :

$$
\left\{\begin{array}{l}
Y_{t}=C Y+H(Y)+F(Y), t>0 \\
Y(0)=Y_{0}=\left(u_{0}, u_{1}\right)^{T}
\end{array}\right.
$$

where

$$
\begin{gathered}
Y=(u, v)^{T}, Y_{0}=\left(u_{0}, u_{1}\right)^{T} \in E, H(Y)=(0,-h(v))^{T}, F(Y)=(0,-f(u)+g)^{T}, \\
C=\left(\begin{array}{cc}
0 & I \\
-A & 0
\end{array}\right), \quad D(C)=D(A) \times H_{0}^{1}(\Omega) .
\end{gathered}
$$

Clearly, $C$ is an unbounded closed operator and generates a $C_{0}$-semigroup on $E$. It is easy to verify by (4)-(5) that $H(Y)+F(Y): E \rightarrow E$ is globally Lipschitz continuous with respect to $Y$. By the classical theory concerning the existence and uniqueness of the solutions [4], we have following lemma.

Lemma 1. Consider the initial value problem (9) on the Hilbert space E.

(i) For any $Y_{0} \in E$, there exists a unique function $Y(\cdot)=Y\left(\cdot, Y_{0}\right) \in C\left(R_{+} ; E\right)$ such that $Y\left(0, Y_{0}\right)=Y_{0}$ and $Y(t)$ satisfies the integral equation

$$
Y(t)=e^{C t} Y_{0}+\int_{0}^{t} e^{C(t-s)}(H(Y(s))+F(Y(s))) d s .
$$

In this case, $Y(t)$ is called a mild solution of (9). (9).

(ii) If $Y_{0} \in D(C)$, there exists $Y(\cdot) \in C\left(R_{+} ; D(C)\right) \cap C^{1}\left(R_{+} ; E\right)$ which satisfies

(iii) $Y\left(t, Y_{0}\right)$ is jointly continuous in $t$ and $Y_{0}$. 
For any $t \geq 0$, we introduce a map $S(t): Y_{0} \mapsto Y\left(t, Y_{0}\right)$, where $Y\left(t, Y_{0}\right)$ is the mild solution (or solution) of $(9)$; then $\{S(t), t \geq 0\}$ is a continuous semigroup on E.

Lemma 2. The semigroup $\{S(t), t \geq 0\}$ determined by system (9) possesses a global attractor $B$ in $E$.

Proof. See [1] for details.

Lemma 3. Consider the linearized equation of (1)-(3)

$$
\left\{\begin{array}{l}
U_{t t}+h^{\prime}\left(u_{t}\right) U_{t}-\Delta U=f^{\prime}(u) U, x \in \Omega, t \geq 0 \\
\left.U(x, t)\right|_{x \in \partial \Omega}=0, t>0 \\
U(x, 0)=(\xi, \eta)^{T}, x \in \Omega
\end{array}\right.
$$

where $\left\{u(t), u_{t}(t)\right\}$ is a mild solution of (1)-(3). Then (10) is a well-posed problem in $E$, the mapping $S(t)$ defined by (9) is Fréchet differentiable on $E$ for any $t>0$, and its differential at $\varphi=\left(u_{0}, u_{1}\right)^{T}$ is the linear operator on $E,(\xi, \eta)^{T} \mapsto$ $(U(t), V(t))^{T}$, where $(U, V)^{T}$ is the solution of (10).

Proof. It is clear from assumptions (4)-(5) that (10) is a well-posed problem in $E$.

We first consider the Lipschitz property of $S(t)$ on $E$. Let $\varphi_{0}=\left(u_{0}, u_{1}\right)^{T} \in$ $E, \widetilde{\varphi}_{0}=\varphi_{0}+(\xi, \eta)^{T}=\left(u_{0}+\xi, u_{1}+\eta\right)^{T} \in E$. It is known from Lemma 1 that the solution $S(t) \varphi_{0}=\varphi(t)=\left(u(t), u_{t}(t)\right)^{T} \in E, S(t) \widetilde{\varphi}_{0}=\widetilde{\varphi}(t)=\left(\widetilde{u}(t), \widetilde{u}_{t}(t)\right)^{T} \in E$. Since there is a global attractor $B$ for $S(t), t \geq 0$ in $E, \varphi(t), \widetilde{\varphi}(t)$ are uniformly bounded in $E$ for $t \geq 0$.

The difference $\psi=\widetilde{u}-u$ satisfies

$$
\psi_{t t}+h\left(\widetilde{u}_{t}\right)-h\left(u_{t}\right)-\Delta \psi=f(\widetilde{u})-f(u) .
$$

Taking the scalar product of (11) with $\psi_{t}=\widetilde{u}_{t}-u_{t}$ in $L^{2}(\Omega)$, we have

$$
\begin{aligned}
\frac{1}{2} \frac{d}{d t}\left(\left|\psi_{t}\right|^{2}+\|\psi\|^{2}\right) & =\left(-\left(h\left(\widetilde{u}_{t}\right)-h\left(u_{t}\right)\right)+f(\widetilde{u})-f(u), \psi_{t}\right) \\
& \leq-\left(h^{\prime}\left(u_{t}+\vartheta_{1}\left(\widetilde{u}_{t}-u_{t}\right)\right) \psi_{t}, \psi_{t}\right)+\left(f^{\prime}\left(u+\vartheta_{2}(\widetilde{u}-u)\right) \psi, \psi_{t}\right) \\
& \leq \text { by }(4)(5) \\
& \leq k\left(\sqrt{\lambda_{1}}\right)^{-1}\|\psi\| \cdot\left|\psi_{t}\right|-\alpha\left|\psi_{t}\right|^{2} \\
& \leq c_{1}\left(\left|\psi_{t}\right|^{2}+\|\psi\|^{2}\right),
\end{aligned}
$$

where $\vartheta_{1}, \vartheta_{2} \in(0,1), c_{1}>0$, i.e.,

$$
\frac{d}{d t}\left(\left|\psi_{t}\right|^{2}+\|\psi\|^{2}\right) \leq 2 c_{1}\left(\left|\psi_{t}\right|^{2}+\|\psi\|^{2}\right)
$$

So, we have the Lipschitz property

$$
\begin{aligned}
\|\widetilde{\varphi}(t)-\varphi(t)\|_{E}^{2} & =\left|\widetilde{u}_{t}(t)-u_{t}(t)\right|^{2}+\|\widetilde{u}(t)-u(t)\|^{2} \\
& \leq \exp \left(2 c_{1} t\right)\left(|\eta|^{2}+\|\xi\|^{2}\right), \forall t \geq 0 .
\end{aligned}
$$

Consider the difference $\theta=\widetilde{u}-u-U$, with $U$ the solution of the linearized equation (10) of (1)-(3). Obviously,

$$
\theta(0)=\theta_{t}(0)=0
$$


and

$$
\begin{aligned}
\theta_{t t}-\Delta \theta= & f(\widetilde{u})-f(u)-f^{\prime}(u)(\widetilde{u}-u)+f^{\prime}(u) \theta \\
& -\left[h\left(\widetilde{u}_{t}\right)-h\left(u_{t}\right)-h^{\prime}\left(u_{t}\right)\left(\widetilde{u}_{t}-u_{t}\right)\right]-h^{\prime}\left(u_{t}\right) \theta_{t}=q .
\end{aligned}
$$

By the mean value theorem, we have

$$
\begin{aligned}
q= & {\left[f^{\prime}\left(u+\vartheta_{3}(\widetilde{u}-u)\right)-f^{\prime}(u)\right](\widetilde{u}-u)+f^{\prime}(u) \theta } \\
& -\left[h^{\prime}\left(u_{t}+\vartheta_{4}\left(\widetilde{u}_{t}-u_{t}\right)\right)-h^{\prime}\left(u_{t}\right)\right]\left(\widetilde{u}_{t}-u_{t}\right)-h^{\prime}\left(u_{t}\right) \theta_{t}
\end{aligned}
$$

where $\vartheta_{i} \in(0,1), i=3,4$.

Taking the scalar product of each side of (14) with $\theta_{t}$ in $L^{2}(\Omega)$, we find

$$
\begin{aligned}
\frac{1}{2} \frac{d}{d t}\left(\left|\theta_{t}\right|^{2}+\|\left.\theta\right|^{2}\right) & =\left(q, \theta_{t}\right) \\
& \leq \text { by }(4)(5) \\
& \leq\left|\theta_{t}\right|\left(\vartheta_{3} k_{1}|\widetilde{u}-u|^{1+\delta_{1}}+k|\theta|+\vartheta_{4} k_{2}\left|\widetilde{u}_{t}-u_{t}\right|^{1+\delta_{2}}-\alpha\left|\theta_{t}\right|\right) \\
& \leq\left|\theta_{t}\right|\left(\vartheta_{3} k_{1}|\widetilde{u}-u|^{1+\delta_{1}}+k|\theta|+\vartheta_{4} k_{2}\left|\widetilde{u}_{t}-u_{t}\right|^{1+\delta_{2}}\right)
\end{aligned}
$$

i.e.,

$\frac{d}{d t}\left(\left|\theta_{t}\right|^{2}+\|\theta\|^{2}\right) \leq c_{2}\left(\left|\theta_{t}\right|^{2}+\|\theta\|^{2}\right)+c_{3}\left(\| \widetilde{u}(t)-\left.u(t)\right|^{2+2 \delta_{1}}+\left|\widetilde{u}_{t}(t)-u_{t}(t)\right|^{2+2 \delta_{2}}\right)$,

where $c_{2}, c_{3}>0$. From the Gronwall inequality, we obtain

$$
\begin{aligned}
\left|\theta_{t}(t)\right|^{2}+\|\theta(t)\|^{2} & \leq \frac{c_{3}}{c_{2}} \exp \left(c_{2} t\right) \cdot \int_{0}^{t}\left(\|\widetilde{u}(t)-u(t)\|^{2+2 \delta_{1}}+\left|\widetilde{u}_{t}(t)-u_{t}(t)\right|^{2+2 \delta_{2}}\right) d \tau \\
& \leq(\operatorname{with}(12)) \\
& \leq c_{4} \exp \left(c_{5} t\right) \cdot\left[\left(|\eta|^{2}+\|\xi\|^{2}\right)^{1+\delta_{1}}+\left(|\eta|^{2}+\|\xi\|^{2}\right)^{1+\delta_{2}}\right], \forall t \geq 0
\end{aligned}
$$

where $c_{4}, c_{5}>0$, that is,

$$
|\widetilde{\varphi}(t)-\varphi(t)-U(t)|_{E}^{2} \leq c_{4} \exp \left(c_{5} t\right) \cdot\left[\left|(\xi, \eta)^{T}\right|_{E}^{2+2 \delta_{1}}+\left|(\xi, \eta)^{T}\right|_{E}^{2+2 \delta_{2}}\right] ;
$$

therefore,

$$
\frac{|\widetilde{\varphi}(t)-\varphi(t)-U(t)|_{E}^{2}}{\left|(\xi, \eta)^{T}\right|_{E}^{2}} \leq c_{4} \exp \left(c_{5} t\right) \cdot\left[\left|(\xi, \eta)^{T}\right|_{E}^{2 \delta_{1}}+\left|(\xi, \eta)^{T}\right|_{E}^{2 \delta_{2}}\right] \rightarrow 0,
$$

as $(\xi, \eta)^{T} \rightarrow 0$ in $E$. The proof is completed.

\section{Proof of Theorem 1}

Let $\varphi=(u, v)^{T}, v=u_{t}+\varepsilon u$, where $\varepsilon$ is chosen as

$$
\varepsilon=\frac{2 \alpha \lambda_{1} \gamma}{\beta^{2}+4 \lambda_{1}}>0
$$

in which $\gamma$ is defined by (6); then system (1)-(3) can be written as

$$
\varphi_{t}+G(\varphi)=F(\varphi), \varphi(0)=\left(u_{0}, u_{1}+\varepsilon u_{0}\right)^{T},
$$

where $F(\varphi)=(0,-f(u)+g)^{T}, G(\varphi)=\left(\varepsilon u-v,-\Delta u+\varepsilon^{2} u-\varepsilon v+h(v-\varepsilon u)\right)^{T}$. 
Lemma 4. For any orthonormal family of elements of $E,\left\{\left(\xi_{j}, \eta_{j}\right)^{T}\right\}_{j=1}^{\ell}$, we have

$$
\sum_{j=1}^{\ell}\left|\xi_{j}\right|^{2} \leq \sum_{j=1}^{\ell} \lambda_{j}^{-1}
$$

Proof. See Lemma VI.6.3 in [2].

To estimate the Hausdorff dimension of the global attractor $B$ for (16) in $E$, we consider the first variation equation of (16)

$$
\Psi^{\prime}+\Lambda \Psi=F^{\prime}(\varphi) \Psi
$$

with initial condition

$$
\Psi(0)=(\xi, \eta)^{T} \in E
$$

where $\Psi=(U, V)^{T}, \varphi=(u, v)^{T}$ is a solution of $(16)$,

$$
\begin{gathered}
F^{\prime}(\varphi)=\left(\begin{array}{cc}
0 & 0 \\
f^{\prime}(u) & 0
\end{array}\right), \Lambda=\left(\begin{array}{cc}
\varepsilon I & -I \\
A+\varepsilon^{2} I-\varepsilon h^{\prime}(v-\varepsilon u) I & h^{\prime}(v-\varepsilon u) I-\varepsilon I
\end{array}\right), \\
D(\Lambda)=D(A) \times H_{0}^{1}(\Omega) .
\end{gathered}
$$

Since the relation of $S(t)$ with $S_{\varepsilon}(t)$ defined by (16) is made by the reversible transformation $u=u, v=u_{t}+\varepsilon u$, it is easy to show from Lemma 3 that (18)-(19) is a well-posed problem in $E$, the mapping $S_{\varepsilon}(t)$ is Fréchet differentiable on $E$ for any $t>0$, and its differential at $\varphi=\left(u_{0}, u_{1}+\varepsilon u_{0}\right)^{T}$ is the linear operator on $E,(\xi, \eta)^{T} \mapsto(U(t), V(t))^{T}$, where $(U, V)^{T}$ is the solution of (18)-(19).

Lemma 5. For any $\varphi=(u, v)^{T} \in E$,

$$
(\Lambda \varphi, \varphi)_{E} \geq \sigma|\varphi|_{E}^{2}+\frac{\alpha}{2}|v|^{2}
$$

where

$$
\sigma=\frac{4 \lambda_{1} \gamma^{2} \alpha}{\beta^{2}+4 \lambda_{1}+\sqrt{\left(\beta^{2}+4 \lambda_{1}\right)\left(\beta^{2}+4 \lambda_{1}-16 \gamma^{2} \lambda_{1}\right)}},
$$

in which $\gamma$ is chosen as in (6).

Proof. By (20), we have

$$
\begin{aligned}
& (\Lambda \varphi, \varphi)_{E}-\sigma|\varphi|_{E}^{2}-\frac{\alpha}{2}|v|^{2} \\
& =\varepsilon\|u\|^{2}-\varepsilon\left(h^{\prime}(v-\varepsilon u) u, v\right)+\varepsilon^{2}(u, v)+\left(h^{\prime}(v-\varepsilon u) v, v\right) \\
& -\varepsilon(v, v)-\sigma\|u\|^{2}-\sigma|v|^{2}-\frac{\alpha}{2}|v|^{2} \\
& \geq \operatorname{by}(5) \\
& \geq(\varepsilon-\sigma)\|u\|^{2}+\left(\frac{\alpha}{2}-\varepsilon-\sigma\right)|v|^{2}-\frac{\varepsilon(\beta+\varepsilon)}{\sqrt{\lambda_{1}}}\|u\| \cdot|v| .
\end{aligned}
$$


Since

$$
\begin{aligned}
& \frac{\varepsilon^{2}(\beta+\varepsilon)^{2}}{4 \lambda_{1}}-(\varepsilon-\sigma)\left(\frac{\alpha}{2}-\varepsilon-\sigma\right) \\
= & \frac{\beta^{4}+2 \beta \varepsilon^{3}}{4 \lambda_{1}}+\left(\frac{\beta^{2}}{4 \lambda_{1}}+1\right) \varepsilon^{2}-\frac{\alpha}{2} \varepsilon+\frac{\alpha}{2} \sigma-\sigma^{2} \\
= & \frac{\varepsilon}{4 \lambda_{1}}\left[\varepsilon^{3}+2 \beta \varepsilon^{2}-4\left(\frac{1}{2}-\gamma\right) \lambda_{1} \alpha\right]+\left(\frac{\beta^{2}}{4 \lambda_{1}}+1\right) \varepsilon^{2}-\gamma \alpha \varepsilon+\frac{\alpha}{2} \sigma-\sigma^{2} .
\end{aligned}
$$

and from (6), (15), (22), elementary computations show

$$
\begin{aligned}
& \varepsilon^{3}+2 \beta \varepsilon^{2}-4\left(\frac{1}{2}-\gamma\right) \lambda_{1} \alpha \leq 0, \\
& \left(\frac{\beta^{2}}{4 \lambda_{1}}+1\right) \varepsilon^{2}-\gamma \alpha \varepsilon+\frac{\alpha}{2} \sigma-\sigma^{2} \leq 0,
\end{aligned}
$$

by (23), we have

$$
(\varepsilon-\sigma)\left(\frac{\alpha \lambda_{1}}{2}-\varepsilon-\sigma\right) \geq \frac{\varepsilon^{2}(\beta+\varepsilon)^{2}}{4 \lambda_{1}}
$$

Thus, the proof is completed.

Lemma 6. Consider the system (16). Let $\Phi$ denote a set of $\ell$ vectors $\left\{\Phi_{i}\right\}_{i=1}^{\ell}$ which are orthonormal in E. If

$$
\sup _{\Phi \subset E} \sup _{\varphi \in B} \sum_{i=1}^{\ell}\left(\left(-\Lambda+F^{\prime}(\varphi)\right) \Phi_{i}, \Phi_{i}\right)_{E} \leq 0,
$$

then the Hausdorff dimension of the global attractor $B$ is less than or equal to $\ell$, i.e.,

$$
\operatorname{dim}_{H}(B) \leq \ell .
$$

Proof. This is a direct consequence of Theorem V. 3.3, equation (V. 3.47)-(V. 3.49) and identity (VI. 6.24) of [2].

Lemma 7. The Hausdorff dimension $d_{H}(B)$ of $B$ for system (16) in E satisfies

$$
d_{H}(B) \leq \min \left\{\ell \mid \ell \in N, \frac{1}{\ell} \sum_{j=1}^{\ell} \lambda_{j}^{-1} \leq \frac{2 \alpha \sigma}{k^{2}}\right\} .
$$

Proof. Let $\ell \in N$ be fixed. Consider $\ell$ solutions $\Psi_{1}, \Psi_{2}, \cdots, \Psi_{\ell}$ of (18)-(19). At a given time $\tau$, let $Q_{\ell}(\tau)$ denote the orthogonal projector in $E$ onto the space spanned by $\Psi_{1}, \Psi_{2}, \cdots, \Psi_{\ell}$. Let $\Phi_{j}(\tau)=\left(\xi_{j}, \eta_{j}\right)^{T} \in E, j=1,2, \cdots, \ell$, be an orthonormal basis of $Q_{\ell}(\tau) E$. From (21) and $\left|\Phi_{j}\right|_{E}=1$, we have

$$
-\left(\Lambda \Phi_{j}, \Phi_{j}\right)_{E} \leq-\sigma-\frac{\alpha}{2}\left|\eta_{j}\right|^{2}
$$

and

$$
\left(F^{\prime}(\varphi) \Phi_{j}, \Phi_{j}\right)_{E}=\left(f^{\prime}(u) \xi_{j}, \eta_{j}\right) \leq \text { by }(4) \leq k\left|\xi_{j}\right| \cdot\left|\eta_{j}\right| \leq \frac{k^{2}}{2 \alpha}\left|\xi_{j}\right|^{2}+\frac{\alpha}{2}\left|\eta_{j}\right|^{2}
$$


Hence, by (17), (26) and (27),

$$
\sum_{i=1}^{\ell}\left(\left(-\Lambda+F^{\prime}(\varphi)\right) \Phi_{i}, \Phi_{i}\right)_{E} \leq-\frac{\ell k^{2}}{2 \alpha}\left(\frac{2 \alpha \sigma}{k^{2}}-\frac{1}{\ell} \sum_{j=1}^{\ell} \lambda_{j}^{-1}\right)
$$

If

$$
\frac{1}{\ell} \sum_{j=1}^{\ell} \lambda_{j}^{-1} \leq \frac{2 \alpha \sigma}{k^{2}}
$$

then

$$
\sum_{i=1}^{\ell}\left(\left(-\Lambda+F^{\prime}(\varphi)\right) \Phi_{i}, \Phi_{i}\right)_{E} \leq 0 .
$$

By Lemma 6, the proof is completed.

Combining with Lemma 7 and (22), we complete the proof of Theorem 1.

\section{Proof of Theorem 2}

We consider the system (1)-(3). If the damping is linear, i.e., $h(v)=\alpha v$, then the equation (1) is

$$
u_{t t}+\alpha u_{t}-\Delta u+f(u)=g, x \in \Omega, t>0 .
$$

Let $\varphi=(u, v)^{T}, v=\dot{u}+\varepsilon_{0} u$, where $\varepsilon_{0}$ is chosen as

$$
\varepsilon_{0}=\frac{\lambda_{1} \alpha}{\alpha^{2}+4 \lambda_{1}} ;
$$

then (29) can be written as

$$
\begin{gathered}
\dot{\varphi}+\Lambda_{0} \varphi=F(\varphi) \\
F(\varphi)=\left(\begin{array}{c}
0 \\
-f(u)+g
\end{array}\right), \quad \Lambda_{0}=\left(\begin{array}{cc}
\varepsilon I & -I \\
A-\varepsilon(\alpha-\varepsilon) I & (\alpha-\varepsilon) I
\end{array}\right) .
\end{gathered}
$$

Lemma 8. For any $\varphi=(u, v)^{T} \in E$,

$$
\left(\Lambda_{0} \varphi, \varphi\right)_{E} \geq \sigma_{0}|\varphi|_{E}^{2}+\frac{\alpha}{2}|v|^{2},
$$

where

$$
\sigma_{0}=\frac{\lambda_{1} \alpha}{\sqrt{\alpha^{2}+4 \lambda_{1}}\left(\alpha+\sqrt{\alpha^{2}+4 \lambda_{1}}\right)} .
$$

Proof. Since

$$
\begin{aligned}
& \left(\Lambda_{0} \varphi, \varphi\right)_{E}-\sigma_{0}|\varphi|_{E}^{2}-\frac{\alpha}{2}|v|^{2} \\
& =\left(\varepsilon_{0}-\sigma_{0}\right)\|u\|^{2}+\left(\frac{\alpha}{2}-\varepsilon_{0}-\sigma_{0}\right)|v|^{2}-\varepsilon_{0}\left(\alpha-\varepsilon_{0}\right)(u, v) \\
& \geq\left(\varepsilon_{0}-\sigma_{0}\right)\|u\|^{2}+\left(\frac{\alpha}{2}-\varepsilon_{0}-\sigma_{0}\right)|v|^{2}-\frac{\varepsilon_{0} \alpha}{\sqrt{\lambda_{1}}}\|u\| \cdot|v|
\end{aligned}
$$

and simple computation by (30) and (34) shows

$$
4\left(\varepsilon_{0}-\sigma_{0}\right)\left(\frac{\alpha}{2}-\varepsilon_{0}-\sigma_{0}\right)=\frac{\varepsilon_{0}^{2} \alpha^{2}}{\lambda_{1}} .
$$

Thus, the proof is completed. 
We consider the first variation equation of (31)

$$
\Psi^{\prime}=-\Lambda_{0} \Psi+F^{\prime}(\varphi) \Psi, \Psi(0)=(\xi, \eta)^{T} \in E,
$$

where $\Psi=(U, V)^{T} \in E$, and $\varphi=(u, v)^{T}$ is a solution of (31)-(2)-(3) and $F^{\prime}(\varphi)$ is defined by (20).

Lemma 9. The Hausdorff dimension $d_{H}\left(B_{1}\right)$ of $B_{1}$ for system (31)-(2)-(3) in $E$ satisfies

$$
d_{H}\left(B_{1}\right) \leq\left\{\ell \mid \ell \in N, \frac{1}{\ell} \sum_{j=1}^{\ell} \lambda_{j}^{-1} \leq \frac{2 \alpha \sigma_{0}}{k^{2}}\right\} .
$$

Proof. Similar to the proof of Lemma 7.

Combining with Lemma 9, (34) and (36), we complete the proof of Theorem 2.

\section{REFERENCES}

[1] J. K. Hale, Asymptotic Behavior Of Dissipative Systems, Amer. Math. Soc., Providence, Rhode Island, 1988. MR 89g:58059

[2] R.Temam, Infinite-dimensional Dynamical Systems in Mechanics and Physics, Appl. Math. Science 68, Springer-Verlag, New York, 1988. MR 89m:58056

[3] G. Wang and S. Zhu, On dimension of the global attractor for damped sine-Gordon equation, Preprint, to appear in J. Math. Phys.

[4] A. Pazy, Semigroups of Linear Operators and Applications to Partial Differential Equations, Appl. Math. Sci., Vol. 44, Springer-Verlag, 1983. MR 85g:47061

Department of Mathematics, Sichuan Union University, Chengdu, 610064, People's Republic of China

E-mail address: nic2601@scuu.edu.cn 